\title{
Evolutionary dynamics of microbial communities in bioelectrochemical systems.
}

\section{Lukasz Szydlowski | Anatoly Sorokin | Olga Vasieva | Susan Boerner |Veyacheslav Fedorovich | 156 \\ Igor Goryanin}

3

Institute of Cell Biophysics, Russia

4

Institute of Physics and Technology, Russia

University of Liverpool, United Kingdom

The School of Informatics, University of Edinburgh, United Kingdom

6

Tianjin Institute for Industrial Biotechnology, China 


\section{SUMMARY}

Bio-electrochemical systems can generate electricity by virtue of mature microbial consortia that gradually and spontaneously optimize performance. To evaluate selective enrichment of these electrogenic microbial communities, five, 3-electrode reactors were inoculated with microbes derived from rice wash wastewater and incubated under a range of applied potentials. Reactors were sampled over a 12-week period and DNA extracted from anodal, cathodal, and planktonic bacterial communities was interrogated using a custom-made bioinformatics pipeline that combined $16 \mathrm{~S}$ and metagenomic samples to monitor temporal changes in community composition. Some genera that constituted a minor proportion of the initial inoculum dominated within weeks following inoculation and correlated with applied potential. For instance, the abundance of Geobacter increased from 423-fold to 766-fold between $-350 \mathrm{mV}$ and $-50 \mathrm{mV}$, respectively. Full metagenomic profiles of bacterial communities were obtained from reactors operating for 12 weeks. Functional analyses of metagenomes revealed metabolic changes between different species of the dominant genus, Geobacter, suggesting that optimal nutrient utilization at the lowest electrode potential is achieved via genome rearrangements and a strong inter-strain selection, as well as adjustment of the characteristic syntrophic relationships. These results reveal a certain degree of metabolic plasticity of electrochemically active bacteria and their communities in adaptation to adverse anodic and cathodic environments. 


\section{1 | INTRODUCTION}

Bio-electrochemical Systems (BESs) refer to microbial communities that either generate electricity, as in Microbial Fuel Cells (MFCs), or utilize electricity, as in Microbial Electrolysis Cells (MECs) (Santoro et al., 2017; Rittmann and Asce, 2017). BES is a well-known technology allowing simultaneous wastewater treatment and electricity production. BES performance depends on activities of electrode-associated bacteria (EAB) that form biofilms on anodal surfaces (e.g. Allen and Bennetto, 1993). Various factors account for $E A B$ enrichment: organic substrates, $\mathrm{pH}$, temperature, electrode composition, and electrical potential (Logan et al., 2006; Aelterman et al., 2008; Torres et al., 2009; Dennis et al., 2016; Rittmann and Asce, 2017).

EAB reach maximum power density when reactors operate at near-neutral $\mathrm{pH}$, at ambient temperatures $\circ$

(25-40 C), and are fed with acetate. Although some studies indicate optimum electrode potentials at ca. $0.3 \mathrm{mV}$ in comparison with a standard hydrogen electrode (SHE) (Aelterman et al., 2008), based upon thermodynamics of acetate consumption, others have found that the most efficient EAB prefer lower anode potentials (Torres et al., 2009). Microbial communities used to inoculate BESs were derived mainly from sludge from wastewater treatment plants (Torres et al., 2009; Ishii et al., 2013; Paitier et al., 2017), aquatic sediments (Holmes et al., 2004), biogas digestate (Daghio et al., 2015), and various environmental samples (e.g. Yates et al., 2012; leropoulos et al. 2010). EAB are abundant in many environments, such that virtually all environmental inocula can eventually give rise to stable EAB consortia within 60 days (Yates et al., 2012). However, further changes and details of community structure are not well understood.

Previous studies analyzed changes within microbial populations for up to several weeks (reviewed in Daghio et al., 2015; Philips et al., 2015; Khater et al., 2017), and employed mainly 16 S sequencing (Ishii et al., 2013; Ishii et al., 2014; Dennis et al., 2016), Ribosomal Intergenic Spacer Analysis (RISA) (Paitier et 
al., 2017), or Denaturing Gradient Gel Electrophoresis (DGGE) (Beecroft et al., 2012). In another study, community shifts were tracked during 90-days of operation (Beecroft et al., 2012). However, those reactors were fed with sucrose, which cannot be metabolized as efficiently (e.g. Schroder, 2007) as acetate (Bond et al., 2002; Bond and Lovley, 2003; Logan et al., 2006; Fedorovich et al., 2009; Daghio et al., 2015). Sucrose-fed systems developed fermentative communities that did not participate in electron transfer. However, these population studies may significantly underestimate microbial diversity, with novel taxonomic groups not detected due to low compatibility with universal primers (e.g. Poretsky et al., 2014; Roselli et al., 2016). Moreover, the type of inoculum also influences community development. In our previous work (Khylias et al., 2015), electrogenic communities derived from different sources exhibit different properties in terms of COD consumption, as well as coulombic efficiencies. Therefore, communities present in particular waste streams should already contain some electrogenic bacterial taxa. The minimal number of EAB is unknown and it remains unclear whether conclusions drawn by Yates et al. (2012) are valid for all inocula. Thus, complex studies examining long-term community changes across a range of $\mathrm{EAB}$-selective electrode potentials have not been attempted.

In this study, we investigated enrichment of EAB from rice wash inoculum in single-chamber, threeelectrode BES. Normalization of conditions among reactors utilizing acetate feeding provided selection pressure for metabolic pathways relevant to electrogenic respiration. Application of potentials ranging from $-50 \mathrm{mV}$ to $-350 \mathrm{mV}$ vs $\mathrm{Ag} / \mathrm{AgCl}(147 \mathrm{mV}$ to $-153 \mathrm{mV}$ vs SHE) on anodes and compositional and functional changes in the resulting bacterial communities were tracked using detailed metagenomics.

\section{2 | RESULTS}

\section{1 | Conditions within reactors}

The single-chamber, 3-electrode BES reactors (M1-M4) were connected to a potentiostat to apply fixed 
potentials (147 mV to $-153 \mathrm{mV}$ vs SHE) to the working electrodes (anodes). We also measured potentials observed (792 mV to $1182 \mathrm{mV}$ vs SHE) on counter electrodes (cathodes). In addition, we prepared one reactor (M5) with only one set of electrodes to operate under open circuit potential (OCP) (Table 1). Our BESs were designed to develop electroactive biofilms, with high volume-to-electrode surface ratios in order to minimize nutrient limitations. Thus, after setting a constant potential, the potentiostat maintained current flow automatically and we did not measure current. One channel of the potentiostat, attached to reactor M3, exhibited an overload error after 8 weeks of operation, resulting in turbidity. This caused a shift in community structure (Fig. S2); hence, we excluded this reactor from our analysis.

\begin{tabular}{|l|r|r|}
\hline Reactor & Anode potential $\mathbf{m V}$ (vs SHE) & \multicolumn{1}{c|}{ Cathode potential $\mathbf{m V}$ (vs SHE) } \\
\hline M1 & $-50(147)$ & $595(792)$ \\
\hline M2 & $-150(47)$ & $775(972)$ \\
\hline M3* & $-250(-53)$ & $885(1082)$ \\
\hline M4 & $-250(-153)$ & $985(1182)$ \\
\hline M5 & 0 to $-530(0 \text { to }-333)^{\mathrm{a}}$ & NA \\
\hline
\end{tabular}

Table 1. Potentials (measured with $\mathrm{Ag} / \mathrm{AgCl}$ reference electrode in saturated $\mathrm{KCl} ;+0.197 \mathrm{mV}$ vs SHE) vs. SHE on anode $(\mathbf{A})$ and cathode $(\mathbf{C})$ of each reactor. ${ }^{\text {a) }}$ Electrode potential measured in the control (OCP) reactor after every 2 weeks. * Around week 8, we experienced a technical fault in M3, resulting in overcharge of the reactor, which caused a shift in the microbial community; results for this reactor are in supplementary material, Fig. S2.

\subsection{Community analysis}

Samples were collected every two weeks from anodes (M1-5A), cathodes (M1-4C) and planktonic (free swimming) fractions (M1-5P). Microscopic imaging (Fig.1) revealed that anodes from M1-M4 reactors 
bioRxiv preprint doi: https://doi.org/10.1101/725580; this version posted November 7, 2019. The copyright holder for this preprint (which was not certified by peer review) is the author/funder, who has granted bioRxiv a license to display the preprint in perpetuity. It is made available under aCC-BY-NC-ND 4.0 International license.

developed communities that formed thick biofilms (Fig. 1a-c), and they were more abundantly

populated than cathodes (Fig. 1e-g). No comparable community formed on electrode strips in M5 (Fig.

1d), due to its OCP mode. Negative charge accumulation on the electrode eventually inhibited microbial growth (Table 1).
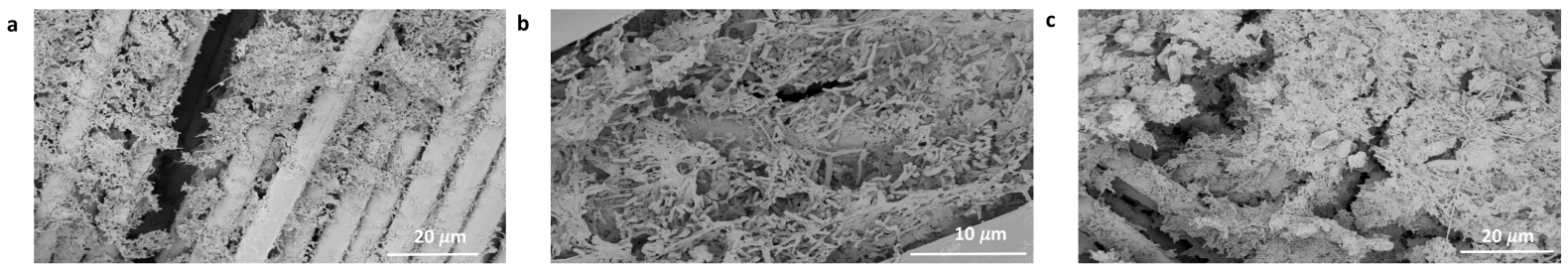

d
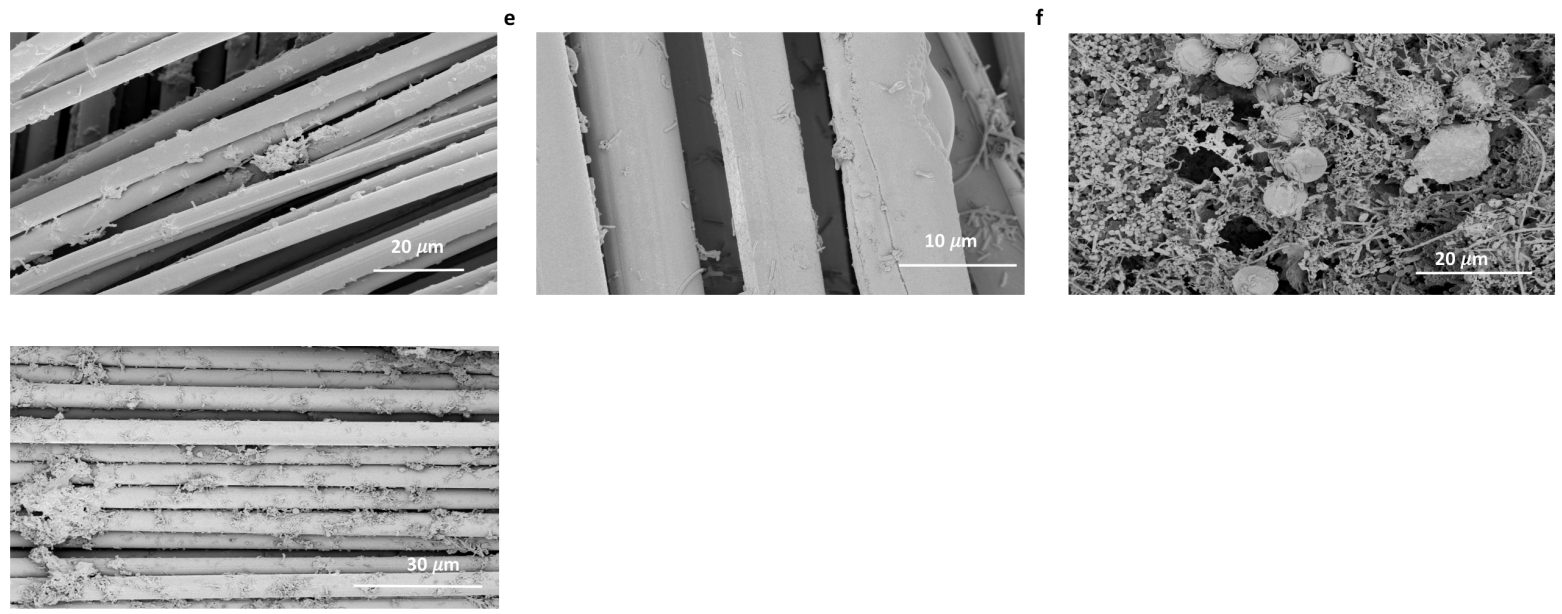

Fig. 1. SEM images of electrodes from a) $M 1 A$, b) $M 2 A$, c) $M 4 A$ d) $M 5 A$ e) $M 1 C$, f) $M 2 C$ and g) $M 4 C$ after 12 weeks of operation. Scale bars are included. 


\section{3 | Taxonomic analysis of anodes}

We analyzed organismal abundances on each anode (Fig.2) during the 12-week operation and compared them with initial community compositions (Table S2). Although rice inoculum is nutrient-rich, the abundance of electrogenic taxa were very low, with Geobacter and Shewanella spp., two of the most efficient EABs, comprising less than $0.09 \%$ and $0.02 \%$ of the total communities, respectively. Enrichment data indicated that the abundance of Geobacter spp. rapidly increased over the first 6-8 weeks under poised anode potential (M1-4). Between 8 and 12 weeks, Geobacter growth rates plateaued and the change in abundance of Geobacter after 12 weeks was 766-fold, 598-fold and 423-fold in M1A, M2A and M4A, respectively, whereas in M5A it only increased 1.2-fold. The relative abundance of Geobacter was significantly higher (ANOVA, $p<0.05$ ) in M1A at $-50 \mathrm{mV}$ and decreased with decreasing potential. After 12 weeks of operation, the abundance of Shewanella remained unchanged in all reactors. Control reactor M5 showed an oscillating pattern of the most abundant genera Methanosaeta, Methanobacterium, and Acinetobacter, with an opposite oscillation pattern of Prevotella (Fig.2d), although the changes are not as significant as in the other reactors. With regard to generic abundance differences between the reactors, for M1A, Geobacter was the only genus with an abundance over $1 \%$ after 12 weeks' operation. M2A had 2 such genera (Geobacter and Denitrovibrio) and in reactor M4 and M5 anodes, the number of significantly abundant (i.e. $>1 \%$ ) genera reached 10 . In the initial inoculum, 5 genera showed abundances $>1 \%$. 

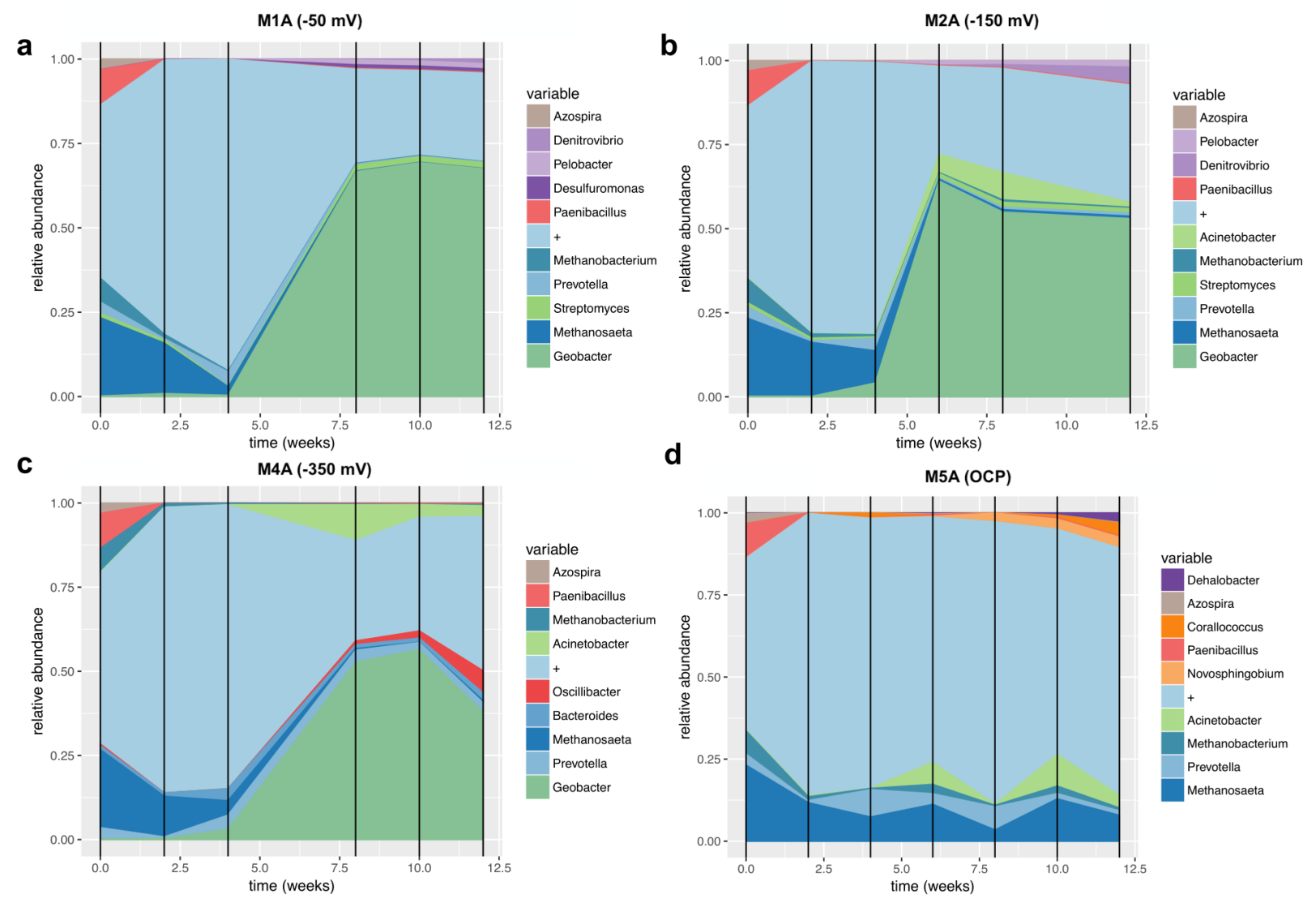

Fig. 2. Relative abundances of anodic dominant genera collected from a) $M 1 A, b) M 2 A, c) M 4 A$ and d) $M 5 A$ during the experiment. Colors represent specific taxonomic groups, "+" refers to all other organisms.

\section{4 | Taxonomic analysis of cathodes}

On the M1 and M2 cathodes, the most abundant organisms were methanogenic archaea (Fig.3a-b);

however, a proportion of Methanosaeta, the most abundant methanogenic genus from the initial inoculum, decreased during the course of the experiment with the subsequent growth of Methanobacter spp. The growth of the latter was in turn inversely correlated with that of Methanoregula. Unexpectedly, Geobacter was the most abundant genus on $\mathrm{M} 4 \mathrm{C}$, reaching a peak abundance of $16 \%, 12$ weeks after inoculation, a level four times higher than that of the next most 
bioRxiv preprint doi: https://doi.org/10.1101/725580; this version posted November 7, 2019. The copyright holder for this preprint (which was not certified by peer review) is the author/funder, who has granted bioRxiv a license to display the preprint in perpetuity. It is made available under aCC-BY-NC-ND 4.0 International license.

abundant genus, Methanobacterium (Fig.3c). Geobacter remained scarce on the M1 and M2 cathodes (< $0.1 \%)$, not exceeding its abundance in the inoculum. On the M4 cathode, a rapid increase in Geobacter abundance after 10 weeks was accompanied by a concomitant decrease of Acinetobacter from $25 \%$ to $2.5 \%$ (Fig.3c).
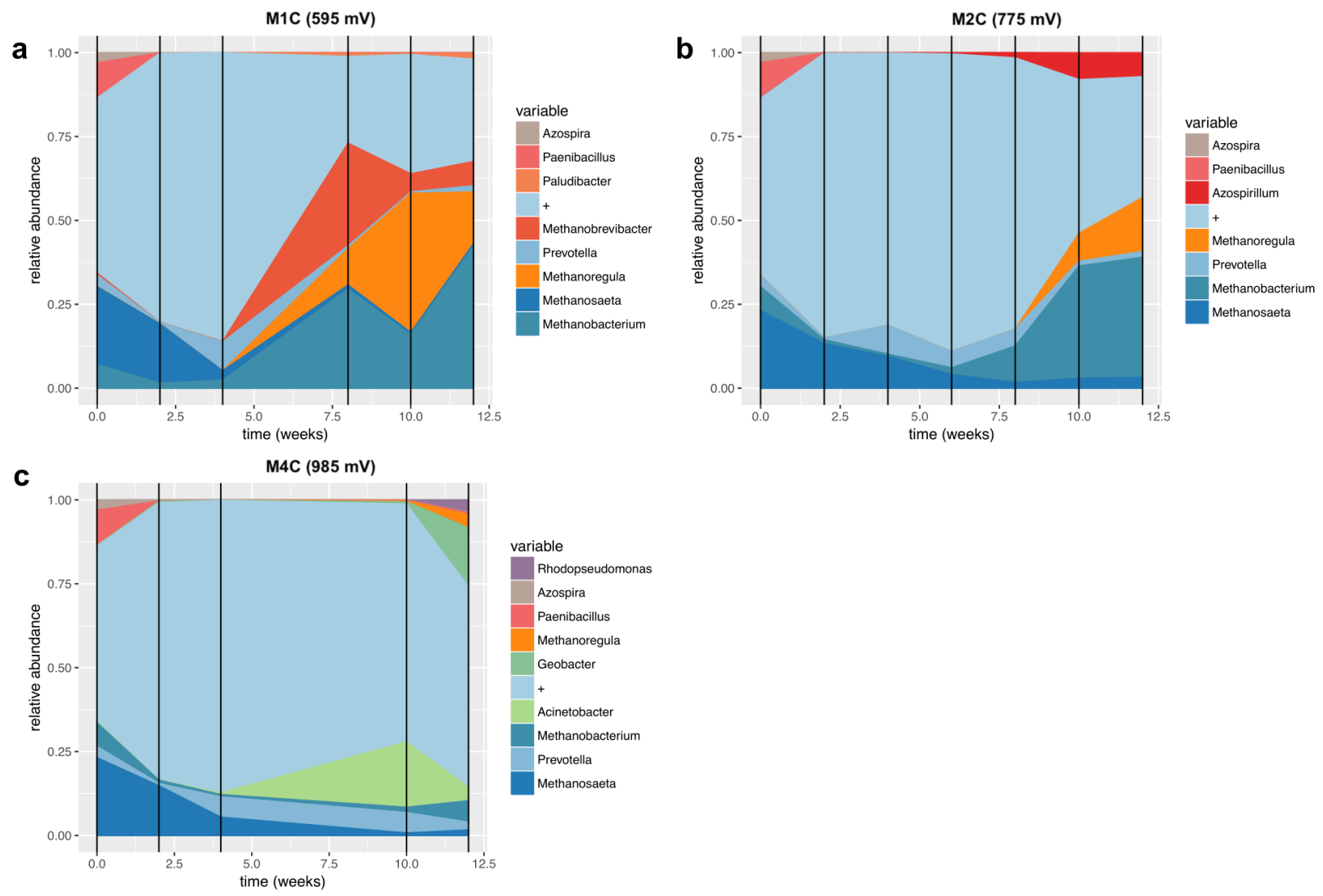

Fig. 3. Relative abundances of cathodic dominant genera collected a) $M 1 C, b) M 2 C$ and c) $M 4 C$ during the experiment. Colors represent specific taxonomic groups, "+" refers to all other organisms.

\section{5 | Taxonomic analysis of planktonic communities}

In the case of planktonic samples (Fig.4), in M1P, a pattern of sudden growth around week 8 , similar to that observed on M1A (although with much lower abundances) was observed with the genera, 
bioRxiv preprint doi: https://doi.org/10.1101/725580; this version posted November 7, 2019. The copyright holder for this preprint (which was not certified by peer review) is the author/funder, who has granted bioRxiv a license to display the preprint in perpetuity. It is made available under aCC-BY-NC-ND 4.0 International license.

Pelomonas, Paludibacter, and Bacteroides. In all planktonic samples, Methanosaeta, the most abundant genus in the inoculum (Table S1), decreased within the first weeks of operation, as did Porphyromonas and Azospirillum. The proportion of Pelomonas, a genus comprising $0.01 \%$ of the initial community, rose to about $20 \%$ of the total planktonic community in M2 after 12 weeks. In M5P, abundances did not reflect the initial community profile, as Methanosaeta decreased within 4 weeks from $24 \%$ to 1\%, whereas Azospirillum abundance reached $\sim 25 \%$. Geobacter abundance was $6.54 \%, 1.92 \%, 4.27 \%$ and $<$ $0.05 \%$ in $M 1, M 2, M 4$ and M5 planktonic communities, respectively.

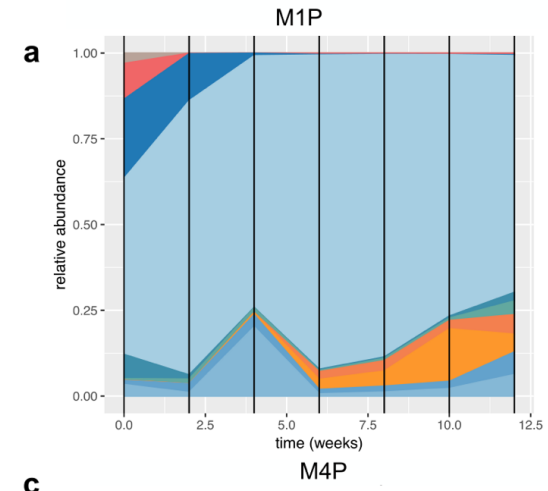

C

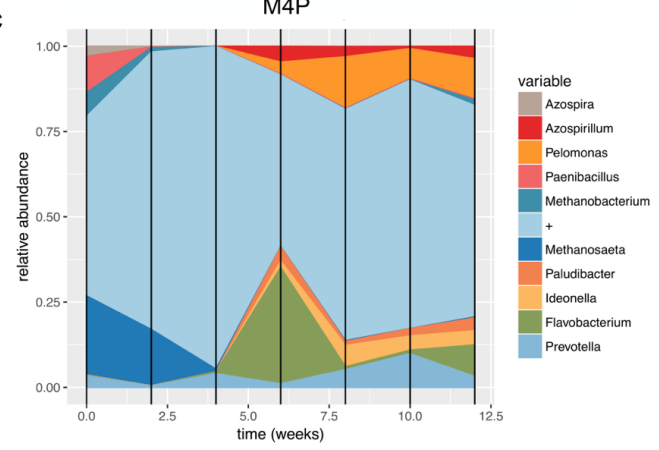

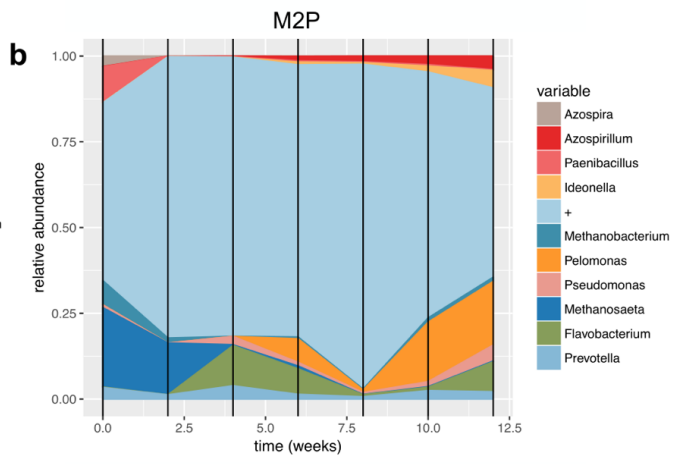

d

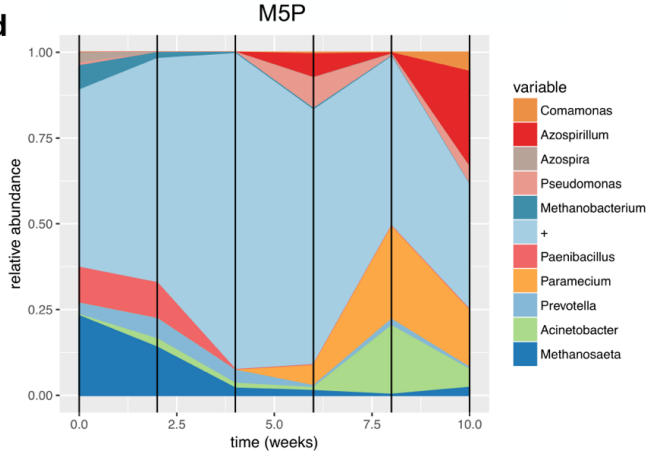

Fig. 4. Relative abundances of planktonic dominant genera collected from a) M1P b) M2P c) M4P and d) M5P during the experiment. Colors represent taxonomic groups, "+" refers to all other organisms.

\section{6 | Diversity of metagenomes}


We used multidimensional scaling to look at how the communities clustered based on sample type (initial sludge, anode, cathode, plankton and OCP) at both the genus level (Fig. 5a) and functional levels (Fig. 5b) after 12 weeks. Significant differences were found for both levels, with $72 \%$ of the distance variability due to sample-type and applied potential at the genus level (Fig. 5a, p-value 0.01), and 65\% (Fig. 5b, p-value 0.04) at the functional level. We also compared the percentage of unclassified reads (at the generic level) from each metagenome to that from the initial inoculum (Fig. S3). Results indicate almost a 2-fold increase of unclassified taxa after 12 weeks in all sampled metagenomes, with the highest being reported in M4P (34.9\%), followed by M1P (31.9\%) and M5P (30.0\%), with $17 \%$ of unclassified genera in the initial inoculum.

a

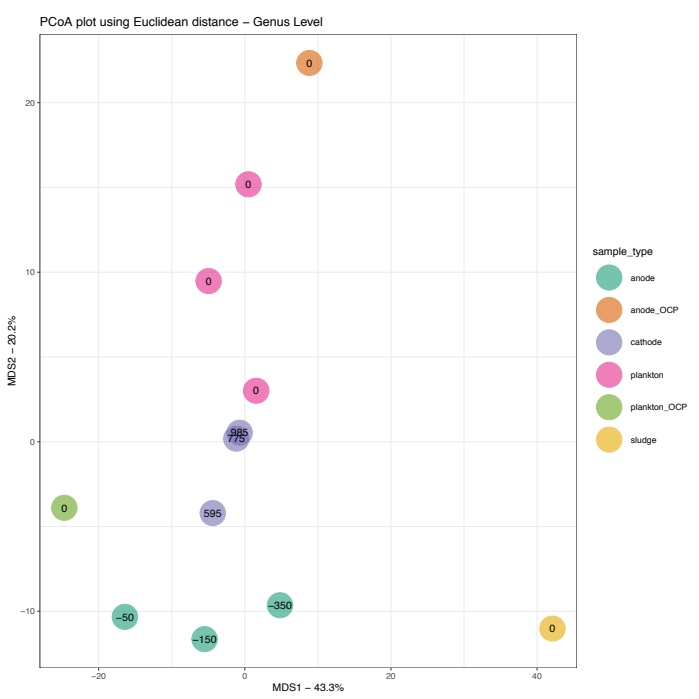

b

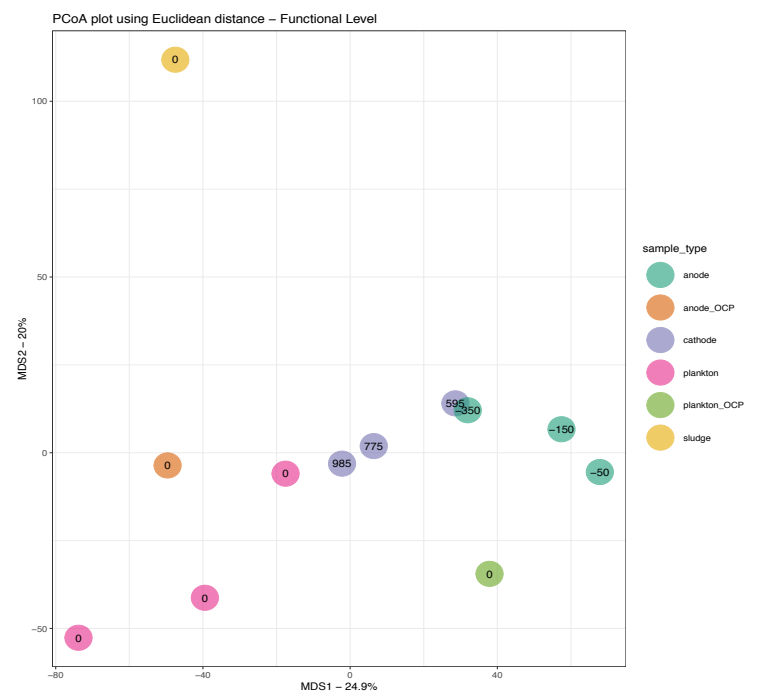

Figure 5. PCoA plots based on a) taxonomy b) function.

\section{7 | Functional overview of metagenomes}

Metagenomic analysis also revealed changes in abundances of functions mapped to genomes 
that were identified via PALADIN analysis. Methanosaeta functions were predominant in the initial communities and the M5 reactor, where they ranked in the top 200 by read count (Tables S3 and S7, respectively). Geobacter functions dominated the ranked lists for the $\mathrm{M} 1, \mathrm{M} 2$, and M4 reactors. To gain a deeper understanding of the communities, we compared abundances in greater detail and ranked the top 200 Geobacter functions from each reactor (Supplementary tables S3-S7). The rank of each gene was established in relation to the normalized abundance of its mapped reads (See Methods for a gene/function abundance calculation) for each species. The function with the highest number of mapped reads was assigned a rank of 1 . Functions with lower numbers of mapped reads had lower ranks with larger assigned values. The main abundance trend defined by the taxonomic analysis, correlates with the occurrence of Geobacter spp. in reactors, with counts for almost all mapped genes decreasing in the order M1 $>M 4>M 2>M 5$. However, we also noticed changes in ranks of several G. metallireducens and G. sulfurreducens genes that may reflect changes in the proportion of these functionally significant genes in Geobacteraceae populations in different reactors. A comparison of M4A to $M 1 A$ revealed that 14 genes increase and 17 genes decrease in rank in M4A with respect to M1A (Fig. 6). An increase in rank order suggests potentially favorable genomic changes and may help to identify species-specific significant functions for specific reactor conditions. The comparative nature of the analysis also helps to avoid biases caused by gene length due to translation of the number of reads into a gene presentation ratio. Interestingly, the results of such a comparison of Geobacter fractions at the M1 and M4 metagenomes (Fig. 6) suggests a positive selection of bacteria for functions/genes involved in electrogenic metabolism. Metagenomic changes in G. sulfurreducens were related to genes encoding ATP synthase, 
$\mathrm{NADH}$-quinone oxidoreductase, and the acetate utilization pathway, with 2-fold, 1.8-fold, and 1.5-fold rank increases in M4A compared to M1A. Conversely, G. sulfurreducens genes encoding ATPase $(p r k A)$, citramalate synthase $(\operatorname{cim} A)$, sodium symporter $(a p / C)$, aldehyde dehydrogenase (aldh), and Fe-S binding protein increased 4-fold, 2.02-fold, 2-fold, 1.52-fold, and 1.51-fold in rank in $\mathrm{M} 1 \mathrm{~A}$, respectively. Changes in the $G$. metallireducens metagenome included a wide range of functions involved in conductive pilin assembly $(p i / B)$, flagella biosynthesis regulation (fgrM), pyruvate metabolism (leuA), electron transfer (nuoB/C/G/L and por), utilization of ammonia (carb-1) efflux pump (cusA), and aspartokinase (asd-1) between 2-fold and 1.5-fold in M4A, whereas periplasmic Ni-Fe dehydrogenase ( $h y b L$ ) and NADH-quinone oxidoreductase (nuoD) show 1.95 and 1.76-fold increase in M1A.

\section{M4A:M1A gene rank}

$\square$ G. metallireducens
$\square$ G. sulfurreducens
$\square$ G. pickeringii
G. uraniireducens
$\square$ G. thiogenes
G. soli
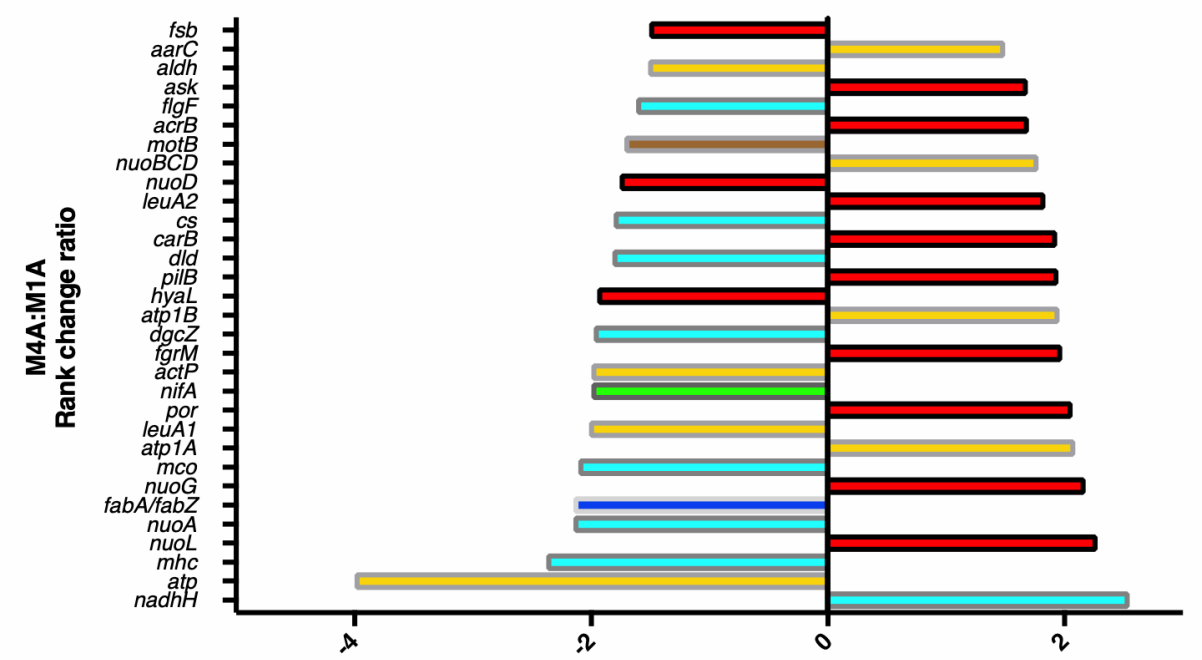

Figure 6. Rank change ratio between M4A and M1A. Colours represent hits with the highest match to one of Geobacter spp. Fold change ratio 1.5 was chosen as a threshold. Represented genes are as follows: $f \boldsymbol{s} b$, Fe-S binding protein; aarC, Succinyl:acetate coenzyme A transferase ; aldh, aldehyde dehydrogenase; ask, aspartokinase; flgF, flagellar basal-body rod protein; $\boldsymbol{a c r} \boldsymbol{B}$, Efflux pump, RND family, inner membrane protein; motB, flagellar basal body stator protein; nuoBCD, NADH-quinone oxidoreductase subunit B/C/D (EC 1.6.5.11); IeuA2, 2-isopropylmalate synthase (EC 2.3.3.13); cs, type I citrate synthase (EC 2.3.3.1); carB, Carbamoylphosphate synthase; dld, Dihydrolipoyl dehydrogenase (EC 1.8.1.4); pilB, Type IV pilus biogenesis ATPase; hyaL, 
Periplasmically oriented, membrane-bound [NiFe]-hydrogenase; atp1B, ATP synthase subunit beta (EC 3.6.3.14); $\boldsymbol{d g} \boldsymbol{c} Z$, Diguanylate cyclase; fgrM, Flagellar biogenesis master sigma-54-dependent transcriptional regulator; $\boldsymbol{a c t} \boldsymbol{P}$, Sodium/solute symporter family protein; nifA, Nif-specific regulatory protein; por, Pyruvate-flavodoxin oxidoreductase; leuA1, (R)-citramalate synthase (EC 2.3.1.182); atp1A, ATP synthase subunit alpha; mco, multicopper oxidase; nuoG, NADH dehydrogenase I, G subunit; fabA/fabZ, Beta-hydroxyacyl dehydratase; nuoA, NADH-ubiquinone oxidoreductase subunit 3; nuoL, NADH dehydrogenase, subunit L; $\boldsymbol{m h}$ c, multiheme cytochrome; atp, ATPase; nadhH, NADH-quinone oxidoreductase subunit H (EC 1.6.5.11).

Genes from the Geobacteraceae (G. pickeringii, G. uraniireducens, G. thiogenes, and G. soli) mostly increased in rank in M1A, with outer membrane multiheme cytochrome c (omc), NADHubiquinone oxidoreductase (nuoA), $\beta$-hydroxyacyl dehydratase (fabA/Z), multicopper oxidase (ompB), Nif-regulatory protein (nifA) exhibiting more than a 2-fold increase in rank, as well as dihydrolipoyl dehydrogenase (IpdA), type I citrate synthase $(g / t A)$, and flagellar components (motB, flgEF), which exhibited 1.82, 1.81 and 1.7-fold shifts in rank, respectively. In M4A, a 2.55-fold rank increase was observed for the NADH quinone oxidoreductase gene $(n u o H)$ from G. pickeringii.

In all planktonic samples, as well as in the initial community, top ranked genes are those involved in genome rearrangement (transposases, reverse transcriptases and endonucleases, see Tables S3-S7), which indicates selective pressure for adaptation to a more competitive environment.

\section{3 | DISCUSSION}

\section{1 | Abundance of Geobacter spp. at anodes is directly proportional to the applied}

\section{voltage}


Geobacter is a well-characterized genus of EAB that populates BES anodes abundantly (Bond and Lovley, 2003). It can comprise $\leq 99 \%$ of bacterial communities isolated from BES electrodes operating at the lowest potential (Torres et al., 2009). However, our work indicates that the abundance of Geobacter increases at anodes with increasing applied potential, meaning that as the electrode potential increases, Geobacter competes more effectively with other genera in the community. In contrast, Shewanella did not increase in abundance under these conditions, likely due to the fact that Shewanella primarily utilizes lactate as a carbon source (e.g. Kim et al., 1999; Pinchuk et al., 2009).

Our abundance results contrast with those of Ishii et al. (2014), in which the highest abundance of Geobacter spp. in acetate-fed, set-potential reactors was observed when anodes were held at $-50 \mathrm{mV}$ vs SHE (-247 $\mathrm{mV}$ vs $\mathrm{Ag} / \mathrm{AgCl})$, whereas in our study the highest abundance was observed on anodes poised at $147 \mathrm{mV}$ vs SHE (-50 mV vs $\mathrm{Ag} / \mathrm{AgCl})$. However, abundances from our study resemble those reported by Dennis et al. (2016), where highest Geobacter abundance was observed on anodes poised at $300 \mathrm{mV}$ vs SHE (103 mV vs $\mathrm{Ag} / \mathrm{AgCl})$. Also, the low initial population of Geobacter in the inoculum (see Table S2) may explain the slower growth of Geobacter spp. at M4 compared to M1 and M2. Although periodic metagenomic sequencing reveals changes in the most abundant genera, it also indicates a large number of potentially undetected bacterial taxa. Changes in this community, as well as interactions among the most abundant $E A B$, will remain enigmatic, however, until genome assembly and isolation methods are improved to identify and characterize new strains. 


\section{2 | Presence of Geobacter spp. at cathodes}

Apart from the Geobacter presence at anodes, Geobacter also dominated the M4 cathode community after 12 weeks, although it was scarcely present at other cathodes $(0.06 \%$ and $0.07 \%$ in $\mathrm{M} 1$ and $\mathrm{M} 2$ cathodes, respectively) as well as at the M5 electrode (0.1\%), being close to the inoculum abundance $(0.08 \%)$. Such an increase in Geobacter spp. abundance in compartments with opposite conditions reflects its ability to both donate and accept electrons in association with electrodes (Holmes et al., 2004; Gregory et al., 2004). However, Geobacter was not found at cathodes in other studies (e.g. Daghio et al., 2015), which may reflect competition with different bacterial taxa, as well as differences in operating conditions, initial community structure, etc. Moreover, rank shifts of flagellar biosynthesis genes demonstrate ongoing colonization of new environmental niches by Geobacter spp. Recently, Rittmann and Asce (2017) concluded that the best-performing EAB has the lowest anode potential, but noted that such conditions are in fact stressful to the bacteria. Perhaps the M4 cathode offered less deleterious conditions for Geobacteriae growth. The continuous decrease of methanogenic archaea at the M4 cathode may hint at competition for electrons with Geobacter spp., a known electrotroph (Strycharz et al., 2011). It is tempting to suggest that incidental oxygen formation, due to the potential difference between M4 electrodes, exceeded the potential difference at which electrolysis of water can occur (1.33 V vs $1.23 \mathrm{~V})$. However, we did not observe bubble formation; therefore, the latter hypothesis may be discarded. Moreover, there are no abundance shifts due to oxygen stress between the M1 and M4 cathodic metagenomes (Table S2), which would certainly follow electrolysis by BES. There is also no evidence of rank shifts in genes involved in hydrogenotrophic methanogenesis, e.g., fumarate reductase, hydrogenase 
within. Microscopic observations (Fig. 1e-g) suggest some other relationship between

Geobacter and methanogens in the cathodic community, for example via direct interspecies

electron transfer (Lovley, 2017).

\subsection{Functional analysis and evidence of differential selection pressure on Geobacter}

\section{at low electrode potentials.}

We were intrigued by the dependence of observed genomic shifts in Geobacter metabolic functions on reactor conditions. The changes themselves, relevant to the main pathways of electrogenic organisms in MFCs, suggest that bacterial genomes evolve rapidly due to metabolic competition. Acetate metabolism is central to the metabolism of $G$. sulfurreducens under electrogenic conditions (Bond and Lovley, 2003; leropoulos et al., 2010). This metabolic feature allows these bacteria to dominate anodic communities if acetate is provided or generated by other members of the syntrophic bacterial community (e.g. G. metallireducens). Interestingly, G. sulfurreducens functions required for acetate utilization (ato) were the only ones from this species that strongly changed rank at lower anodic potential (M4), with aldehyde dehydrogenase rank decreasing (Fig. 6). Acetate utilization by G. sulfurreducens may be supported by a syntrophic association with Pelobacter spp. (Sreshtha et al., 2013), and enrichment of this genus was observed on anodes M1 and M2 (Fig. 2a-b). Though acetate was provided in the medium, local interactions between bacteria and bacterial clusters may be significant. Sequences corresponding to ATP synthase subunits also increased in abundance in M4A, with a 4-fold decrease in ATPase (Fig. 6), suggesting higher pressure for energy generation. 
At the same time, adaptation and evolution of highly electrogenic G. metallireducens at M4 seems to proceed due to a requirement for conductive pili, respiratory NADH dehydrogenases, and pyruvate metabolism, which increase in rank at M4A (Fig. 6). NuoL, for which metagenomic rank changed most at the M4 anode compared to M1A, is responsible for the reverse electron transfer and $\mathrm{H}^{+} / \mathrm{e}^{-}$stoichiometry (Steimle et al., 2011), which may be important for balancing electron flow between NAD+ and ferredoxin pools. PilB is an ATPase required for polymerization of conductive e-pili (McCallum et al., 2017), and pilB mutants are reported to generate lower current and form thinner biofilms (Steidl et al., 2016). Additionally, it was observed by Ishii et al. (2018) that pilA expression increased at lower surface potential. Change in pilB expression has not been reported in the aforementioned study, although it could be a result of a normalization procedure: with an increase in the abundance of DNA reads, changes in abundance of RNA reads/expression could not be observed.

Flagellar response regulator (fgrM), which increased in rank in M4A, regulates flagellar growth, a known feature of G. metallireducens when grown with an insoluble Fe(III) source (Ueki et al., 2012). This feature corresponds to increased motility of cells when Fe(III) sources are sparse. Cells can store electrons in their numerous cytochromes, acting as capacitors, so that they can discharge them upon the next available Fe(III) cluster. Similarly, lower surface potential of the anode in M4A could lead to formation of dispersed local spots for electron release. Thus, $G$. metallireducens cells with regulated expression of flagella proteins could possess higher and more ordered motility, which, together with the higher capacity to polymerize e-pili by PilB, should give them an advantage over competitors.

This study shows a decrease in rank of genes belonging to other taxa (G. pickeringii, G. 
uraniireducens, G. thiogenes and G. soli) at lower surface potential (Fig. 6b). Citrate synthase (G. pickeringii), a proposed indicator of Geobacteraceae metabolic activity (Holmes et al., 2005) decreased almost 2-fold at M4A. Also, genes encoding components of EET are lower in rank at M4A (Fig. 6b). This correlates with the limited capacity of these Geobacteraceae to adapt to low surface potential, as their electrochemical activity was reported for rather more positive redox potentials (Ishii et al., 2018).

We conclude that the comparative rank measurement gives a better estimate of a functional genomic shift for a particular metagenome in relation to a reactor condition, which is not surprising when one takes into account that ranks are known to be a robust characterisation of the population for analysis of covariance (Conover and Iman, 1982; Quade, 1967). The rank transformation has been widely used since Charles Spearman defined the correlation coefficient in 1904 and more recently it has been adapted in metagenomics studies of microbial communities. (e.g. Saeedghalati et al., 2017). Certainly, gene abundance cannot be taken as an unequivocal reflection of activity levels of relevant functions in bacterial metabolism under different conditions. However, we suggest that genomic rearrangements represent responses to specific functional requirements. Genes may be retained or even propagate in a population if they enhance organismal fitness. Increasing abundances of bacterial strains bearing advantageous genes may also explain the observed phenomenon. As shown in the case of pilB gene, analysis of gene ranks derived from metagenomics analysis can complement expression studies.

\section{4 | The importance of unknown organisms in a functional overview of these}




\section{metagenomes}

The presence of unclassified taxa indicates an increase in abundance of unknown organisms upon inoculation into BES reactors. Our results (Fig. S3) do not align with other works (Ishii et al., 2014; Dennis et al., 2016), in which unclassified taxa in the inoculum contained comparable numbers of unclassified reads, but only several percent of unclassified organisms were identified in sampled electrodes. The discrepancy between the two studies may be due to the difference in sampling and sequencing methods. The former studies employed only $16 \mathrm{~S}$ samples, whereas we compared Illumina MiSeq whole metagenomic reads. Such discrepancies were also reported in a later study by Ishii et al. (2018), where lower diversity was reported in the same samples when only $16 \mathrm{~S}$ analysis was employed. The abundance of novel unidentified organisms suggests the existence of novel electrogenic microorganisms. Such organisms may not be as efficient in EET as Geobacter spp.; hence the term "weak electricigens" (Doyle and Marsili, 2018), but they may nonetheless provide useful insight into the divergence of EET mechanisms. However, since their increases do not follow electrode potential, the presence of so many unclassified organisms is perhaps more dependent on the inoculum than the reactor conditions.

\section{4 | EXPERIMENTAL PROCEDURES}

\section{1 | Reactor setup and operating conditions}

Four reactors (M1-4) were designed as follows: $1.2 \mathrm{~L}$ chamber with an anode consisting of 6 carbon-fiber strips (Zoltec) $3 \times 10 \mathrm{~cm}$ connected with titanium wire (Kojundo chemical laboratory), a cathode 
consisting of 6 carbon-fiber strips (Zoltec) $3 \times 10 \mathrm{~cm}$ connected with titanium wire, and a reference electrode (Radiometer Analytical, Hach). Additionally, one control reactor (M5) consisted only of one set of 6 carbon-fiber strips (Zoltec) $3 \times 10 \mathrm{~cm}$ connected with titanium wire and reference electrode (Radiometer Analytical, Hach), and was operated in open circuit mode (see Fig.S1 for schematic view). A four-channel potentiostat (UniChem) was connected to each reactor with stainless steel clips and potential differences of $-50 \mathrm{mV},-150 \mathrm{mV},-250 \mathrm{mV},-350 \mathrm{mV}$ vs Ag/AgCl $(147 \mathrm{mV}, 47 \mathrm{mV},-53 \mathrm{mV}$ and $153 \mathrm{mV}$ vs SHE) were applied to anodes M 1-4A, respectively. Each reactor was inoculated with rice wash water $(1.2 \mathrm{~L})$ and incubated for 2 weeks at room temperature $\left(23^{\circ} \mathrm{C}\right)$, after which the liquid was replaced with an equal volume of the following medium: $0.05 \mathrm{M}$ phosphate buffer (pH 6), $200 \mathrm{mg} / \mathrm{L}$ $\mathrm{CaCl}_{2} \bullet 2 \mathrm{H}_{2} \mathrm{O}, 250 \mathrm{mg} / \mathrm{L} \mathrm{MgCl}_{2} \bullet 6 \mathrm{H}_{2} \mathrm{O}, 500 \mathrm{mg} / \mathrm{L} \mathrm{NH}_{4} \mathrm{Cl}$, sodium acetate $2 \mathrm{~g} \mathrm{COD} / \mathrm{L}$ (Fedorovich et al., 2009). COD concentration was measured using a Hach COD kit (Hach, USA). The medium was replaced 6 times at 2-week intervals, yielding a total operating time of 12 weeks. Additionally, $50 \mathrm{~mL}$ of liquid fraction and one strip of each electrode (1x A and 1x C from M1-4 and 1x A from M5) were collected with every change of medium. These were used for DNA extraction and SEM analysis.

\section{2 | Microscopic imaging}

Samples for microscopic imaging were taken simultaneously with the DNA samples and processed with osmium, as follows (Fischer et al., 2012): upon removal from the anode compartment, the samples were immediately cut by knife, and fixed by $1 \%$ Osmium diluted with $0.2 \mathrm{M}$ Cacodylate (Wako) buffer 30min. The samples were then washed three times with RQ water and dehydrated stepwise with a graded series of ethanol solutions (70, 80, 90, 95 and three times 100\%). The electrode samples were finally critical-point dried with tert-butyl ethanol and sputter coated with a thin layer of gold. The samples were analyzed by a scanning electron microscopy (SEM) (JSM-7900F JEOL). 


\section{3 | DNA extraction and library preparation}

DNA was extracted using TRIzol (Life Technologies) and additional samples were subjected to Maxwell extraction (GMO purefood kit, Maxwell) using an automated RSC system (Promega). Samples with sufficient amounts of DNA were subjected to Illumina sequencing (48). Remaining samples (32) were subjected to $16 \mathrm{~S}$ sequencing. DNA libraries were constructed using Nextera XT kit (Illumina) and sequencing was performed on MiSeq platform (Illumina, San-Diego, CA, USA). Samples were uploaded to MG-RAST (mgp81854 for 16S, mgp82844 for metagenomes). We were unable to collect data from 3 cathodal samples (see Table S1).

\section{4 | Bioinformatic analysis}

Whole-genome sequences and $16 \mathrm{~S}$ sequences were analyzed using a custom-developed pipeline, as described elsewhere (Orakov et al., 2017), which carried out taxonomic analysis using Kaiju (Menzel and Krogh, 2016), as well as functional analysis using PALADIN (only applicable to metagenomes)

(Westbrook et al., 2017). Results of PALADIN analysis for anodes M1 and M4 can be found in the Supplementary material (Table S3). Compositional analysis of communities was performed in R version 1.4.0 (van den Boogaart et al., 2018) with package compositions (van den Boogaart and TolosanaDelgado, 2008). Relative abundance was represented as composition with absolute geometry (rcomp). To combine $16 \mathrm{~S}$ and metagenomic sequences, datasets from Kaiju and MG-RAST were manually curated (a more detailed description can be found at https://github.com/Iptolik/ASAR). One-way ANOVA was conducted to determine the significance of differences in abundance of Geobacter between M1A, M2A and M4A for the period between week 8 and 12. For visualization purposes, the five most abundant genera in the inoculum and five most abundant genera in Week 12 were selected. All other genera were included in the "Other" group. The R script employed is described in Orakov et al. (2017). The analysis of 
metagenome diversity was carried out using R version 3.6.0 (need to add ref here and below).

Multidimensional scaling was performed with the "dist" and "cmdscale" functions, and MDS (PCoA)

plots were generated with ggplot2. For PERMANOVA, we used the "adonis" function in the vegan

package (Anderson, 2001).

\section{ACKNOWLEDGEMENTS}

This research was supported by Okinawa Institute of Science and Technology Graduate University. We thank Dr. Larisa Kiseleva for collecting electrode and plankton samples and Dr. Toshio Sasaki for preparing SEM specimens.

\section{CONFLICT OF INTEREST}

The authors declare no conflict of interest. 


\section{REFERENCES}

Aelterman, P., Freguia, S., Keller, J., Verstraete, W. and Rabaey, K. (2008) The anode potential regulates bacterial activity in microbial fuel cells. Applied microbiology and biotechnology, 78, 409-418.

Allen, R. M. and Bennetto, H. P. (1993) Microbial fuel cells: Electricity production from carbohydrates. Applied Biochemistry and Biotechnology, 39, 27-40.

Anderson, M.J. 2001. A new method for non-parametric multivariate analysis of variance. Australian Ecology, 26: 32--46.

Beecroft, N. J., Zhao, F., Varcoe, J. R., Slade, R. C. T., Thumser, A. E. and Avignone-rossa, C. (2012) Dynamic changes in the microbial community composition in microbial fuel cells fed with sucrose. Applied Microbiology and Biotechnology, 93 423-437.

Bond, D. R., Holmes, D. E., Tender, L. M. and Lovley, D. R. (2002) Electrode-Reducing Microorganisms That Harvest Energy from Marine Sediments. Science, 295, 483-485.

Bond, D. R. and Lovley, D. R. (2003) Electricity Production by Geobacter sulfurreducens Attached to Electrodes Electricity Production by Geobacter sulfurreducens Attached to Electrodes. Applied and Environmental Microbiology, 69, $1548-1555$.

van den Boogaart, K., Tolosana, R. and Bren, M. (2018) Compositions: Compositional data analysis. r-package version 1.40-1.

van den Boogaart, K. G. and Tolosana-Delgado, R. (2008) "compositions": A unified R package to analyze compositional data. Computers \& Geosciences, 34, 320-338.

Brooks, J. P., Edwards, D. J., Harwich, M. D., Rivera, M. C., Fettweis, J. M., Serrano, M. G., Reris, R. A., Sheth, N. U., Huang, B., Girerd, P., Strauss, J. F., Jefferson, K. K. and Buck, G. A. (2015) The truth about metagenomics: Quantifying and counteracting bias in 16S rRNA studies Ecological and evolutionary microbiology. BMC Microbiology, 15, 1-14.

Conover, W.J and Iman, R.L. (1982) Analysis of covariance using the rank transformation. Biometrics, 38(3), 715-724.

Cord-Ruwisch, R., Lovley, D. R., Schink, B. (1998). Growth of Geobacter sulfurreducens with acetate in syntrophic cooperation with hydrogen-oxidizing anaerobic partners. Applied and Environmental Microbiology, 64(6), 2232-2236.

Daghio, M., Gandolfi, I., Bestetti, G., Franzetti, A., Guerrini, E. and Cristiani, P. (2015) Anodic and cathodic microbial communities in single chamber microbial fuel cells. New Biotechnology, 32, 79-84.

Dennis, P. G., Virdis, B., Vanwonterghem, I., Hassan, A., Hugenholtz, P., Tyson, G. W. and Rabaey, K. (2016) Anode potential influences the structure and function of anodic electrode and electrolyte-associated microbiomes. Scientific Reports,6, 1-11.

Doyle, L. E., \& Marsili, E. (2018). Weak electricigens: A new avenue for bioelectrochemical research. Bioresource Technology, $258,354-364$.

Dupuis, A., Peinnequin, A., Darrouzet, E., Lunardi, J. (1997). Genetic disruption of the respiratory NADH-ubiquinone reductase of Rhodobacter capsulatus leads to an unexpected photosynthesis-negative phenotype. FEMS Microbiology Letters, 148(1), 107- 
114.

Fedorovich, V., Knighton, M. C., Pagaling, E., Ward, F. B., Free, A. and Goryanin, I. (2009) Novel electrochemically active bacterium phylogenetically related to Arcobacter butzleri, isolated from a microbial fuel cell. Applied and Environmental Microbiology, 75, 7326-7334.

Fischer, E. R., Hansen, B. T., Nair, V., Hoyt, F. H. and Dorward, D. W. (2012) Scanning Electron Microscopy. Current Protocols in Microbiology, 1-16.

Gregory, K. B., Bond, D. R. and Lovley, D. R. (2004) Graphite electrodes as electron donors for anaerobic respiration. Environmental Microbiology, 6, 596-604.

Holmes, D. E., Bond, D. R., O'Neil, R. A., Reimers, C. E., Tender, L. R. and Lovley, D. R. (2004) Microbial communities associated with electrodes harvesting electricity from a variety of aquatic sediments. Microbial Ecology, 48, 178-190.

leropoulos, I., Winfield, J. and Greenman, J. (2010) Effects of flow-rate, inoculum and time on the internal resistance of microbial fuel cells. Bioresource Technology, 101, 3520-3525.

Ishii, S., Suzuki, S., Norden-Krichmar, T. M., Tenney, A., Chain, P. S. G., Scholz, M. B., Nealson, K. H. and Bretschger, O. (2013) A novel metatransctiptomic approach to identify gene expression dynamics during extracellular electron transfer. Nature Communications, 4:1601, 1-10.

Ishii, S., Suzuki, S., Norden-Krichmar, T. M., Phan, T., Wanger, G., Nealson, K. H., ... Bretschger, O. (2014). Microbial population and functional dynamics associated with surface potential and carbon metabolism. ISME Journal, 8(5), 963-978.

Ishii, S., Suzuki, S., Tenney, A., Nealson, K. H., \& Bretschger, O. (2018). Comparative metatranscriptomics reveals extracellular electron transfer pathways conferring microbial adaptivity to surface redox potential changes. ISME Journal, 12(12), 28442863.

Kim, H. J., Hyun, M. S., Chang, I. S., \& Kim, B. H. (1999). A microbial fuel cell type lactate biosensor using a metal-reducing bacterium, Shewanella putrefaciens. Journal of Microbiology and Biotechnology, 9(3), 365-367.

Pinchuk, G. E., Rodionov, D. A., Yang, C., Li, X., Osterman, A. L., Dervyn, E., ... Beliaev, A. S. (2009). Genomic reconstruction of Shewanella oneidensis MR-1 metabolism reveals a previously uncharacterized machinery for lactate utilization. Proceedings of the National Academy of Sciences, 106(8), 2874-2879.

Khater, D. Z., El-khatib, K. M. and Hassan, H. M. (2017) Microbial diversity structure in acetate single chamber microbial fuel cell for electricity generation. Journal of Genetic Engineering and Biotechnology, 15, 127-137.

Logan, B. E., Hamelers, B., Rozendal, R., Schroder, U., Keller, J., Freguia, S., Aelterman, P., Verstraete, W. and Rabaey, K. (2006) Microbial Fuel Cells: Methodology and Technology. Environmental Science and Technology, 40 (17), 5181-5192.

Lovley, D. R. (2017). Syntrophy Goes Electric: Direct Interspecies Electron Transfer. Annual Review of Microbiology, 71(1), 643- 
664.

McCallum, M., Tammam, S., Khan, A., Burrows, L. L., \& Lynne Howell, P. (2017). The molecular mechanism of the type IVa pilus motors. Nature Communications, 8(May), 1-10.

Menzel, P. and Krogh, A. (2016) Kaiju: Fast and sensitive taxonomic classification for metagenomics. Nature communications, 7, $1-9$.

Orakov, A., Sakenova, N., Sorokin, A. and Goryanin, I. (2017) ASAR: visual analysis of metagenomes in R. Bioinformatics, 0-0.

Orellana, Roberto (2014). "PHYSIOLOGICAL MODELS OF GEOBACTER SULFURREDUCENS AND DESULFOBACTER POSTGATEI TO UNDERSTAND URANIUM REMEDIATION IN SUBSURFACE SYSTEMS" (2014). Doctoral

Dissertations.

Jari Oksanen, F. Guillaume Blanchet, Michael Friendly, Roeland Kindt, Pierre Legendre, Dan McGlinn, Peter R. Minchin, R. B. O'Hara, Gavin L. Simpson, Peter Solymos, M. Henry H. Stevens, Eduard Szoecs and Helene Wagner (2019). vegan:

Community Ecology Package. R package version 2.5-6. https://CRAN.R-project.org/package=vegan

Paitier, A., Godain, A., Lyon, D., Haddour, N., Vogel, T. M. and Monier, J.-M. (2017) Biosensors and Bioelectronics Microbial fuel cell anodic microbial population dynamics during MFC start-up. Biosensors and Bioelectronics, 92,357-363.

Philips, J., Verbeeck, K., Rabaey, K. and Arends, J. (2015) Electron transfer mechanism in biofilms. In Microbial Electrochemical and Fuel Cells Fundamentals and Applications (eds. K. Scott and E. H. Yu), chap. 3, 113-169. Cambridge: Woodhead Publishing (Elsevier), first edn.

Poretsky, R., Rodriguez-r, L. M., Luo, C., Tsementzi, D. and Konstantinidis, K. T. (2014) Strengths and Limitations of 16S rRNA Gene Amplicon Sequencing in Revealing Temporal Microbial Community Dynamics. PLOS One, 9(4), e93827.

Quade, D. (1967) Rank analysis of covariance. Journal of the American Statisctical Association, 62, 1187-1200.

R Core Team (2019). R: A language and environment for statistical computing. R Foundation for Statistical Computing, Vienna, Austria. URL https://www.R-project.org/.

Rosselli, R., Romoli, O., Vitulo, N., Vezzi, A., Campanaro, S., De Pascale, F., ... Squartini, A. (2016). Direct 16S rRNA-seq from bacterial communities: A PCR-independent approach to simultaneously assess microbial diversity and functional activity potential of each taxon. Scientific Reports, 6, 1-12.

Rittmann, B. E. and Asce, D. M. (2017) Ironies in Microbial Electrochemistry. Journal of Environmental Engineering, $143,1-7$.

Saeedghalati, M., Farahpour, F., Budeus, B., Lange, A., Westendorf, A. M., Seifert, M., ... Hoffmann, D. (2017). Quantitative Comparison of Abundance Structures of Generalized Communities: From B-Cell Receptor Repertoires to Microbiomes. PLoS Computational Biology, 13(1), 1-29.

Santoro, C., Arbizzani, C., Erable, B., leropoulos, I. (2017). Microbial fuel cells: From fundamentals to applications. A review. Journal of Power Sources, 356, 225-244. 
Schroder, U. (2007) Anodic electron transfer mechanisms in microbial fuel cells and their energy efficiency. Physical Chemistry

Chemical Physics, 9, 2619-2629.

Shrestha, P. M., Rotaru, A. E., Summers, Z. M., Shrestha, M., Liu, F., Lovley, D. R. (2013). Transcriptomic and genetic analysis of direct interspecies electron transfer. Applied and Environmental Microbiology, 79(7), 2397-2404.

Shrestha, P. M. and Rotaru, A. E. (2014) Plugging in or going wireless: Strategies for interspecies electron transfer. Frontiers in

Microbiology, 5, 1-8.

Steidl, R. J., Lampa-Pastirk, S., \& Reguera, G. (2016). Mechanistic stratification in electroactive biofilms of Geobacter sulfurreducens mediated by pilus nanowires. Nature Communications, 7.

Steimle, S., Bajzath, C., Dorner, K., Schulte, M., Bothe, V., Friedrich, T. (2011). Role of subunit NuoL for proton translocation by respiratory complex I. Biochemistry, 50(16), 3386-3393.

Torres, C. I., Krajmalnik-Brown, R., Parameswaran, P., Marcus, A. K., Wanger, G., Gorby, Y. A. and Rittmann, B. E. (2009)

Selecting Anode-Respiring Bacteria Based on Anode Potential: Phylogenetic, Electrochemical, and Microscopic Characterization.

Environ. Sci. Technol., 43, 9519-9524.

Westbrook, A., Ramsdell, J., Schuelke, T., Normington, L., Bergeron, R. D., Thomas, W. K. and MacManes, M. D. (2017) PALADIN: Protein alignment for functional profiling whole metagenome shotgun data. Bioinformatics, 33, 1473-1478.

Wickham, H. ggplot2: Elegant Graphics for Data Analysis. Springer-Verlag New York, 2016

Yates, M. D., Kiely, P. D., Call, D. F., Rismani-Yazdi, H., Bibby, K., Peccia, J., Regan, J. M. and Logan, B. E. (2012) Convergent development of anodic bacterial communities in microbial fuel cells. ISME Journal, 6, 2002-2013. 